\title{
The role of the ASPL-TFE3 fusion protein in Alveolar Soft Part Sarcoma
}

Shuxin Wang ${ }^{1}$, Amir Pozner ${ }^{2}$, Mary Neison ${ }^{2}$, Kevin Jones ${ }^{3}$ and Peter Shen ${ }^{4}$

${ }^{1}$ Peter Shen/the University of Utah, United States, ${ }^{2}$ Department of Orthopaedics, Department of Oncological Sciences, Huntsman Cancer Institute/the Univerisity of Utah, United States, ${ }^{3}$ Department of Orthopaedics, Department of Oncological Sciences, Huntsman Cancer Institute/the University of Utah, United States, ${ }^{4}$ University of Utah, Salt lake City, Utah, United States

The AAA+ ATPase Valosin-Containing Protein VCP (also known as Cdc48/p97) is a central player in maintaining protein homeostasis. VCP is best described for its role in unfolding ubiquitinated proteins prior to their degradation. Like many other AAA+ ATPases, VCP functions as a homo-hexameric complex and is regulated by interactions with many binding partners [1-4]. One such binding partner is alveolar soft part locus (ASPL), which was shown to destabilize VCP hexamers into VCP-ASPL heterotetramers $[5,6]$. Interestingly, a chromosomal translocation mutation in ASPL causes alveolar soft part sarcoma (ASPS), a rare soft tissue cancer with poor prognosis. The non-reciprocal chromosomal translocation $\mathrm{t}(\mathrm{X} ; 17)(\mathrm{p} 11.2 ; \mathrm{q} 25)$ leads to the formation of a fusion protein between the N-terminal region of the ASPL gene with the C-terminal region of transcription factor E3 (TFE3) [7]. The ASPL-TFE3 fusion protein (hereafter AT3) retains the VCP binding domain of ASPL and the DNA binding, activation domain, and nuclear localization signal domain of TFE3[7-9]. Several studies have shown that AT3 up-regulates various transcripts that are essential for cell proliferation [4,10-11]. However, the relation between VCP and the AT3 fusion protein is poorly understood. The premise of this work is to investigate the structure and function relationship between AT3 and VCP.

The destabilization of VCP by ASPL is caused by C-terminal motifs within ASPL, including an $\alpha$-helical lariat and cis-Pro touch-turn (residues 313-553) [6]. Interestingly, the ASPL portion within the AT3 fusion protein (residues 1-311) lacks these destabilization motifs. Based on these observations, we investigated whether AT3 interacts with intact or destabilized VCP complexes. Co-immunoprecipitation (co-IP) experiments from HEK293T cells transfected with AT3 indicate that AT3 forms stable complexes with VCP hexamers (Fig. 1). Negative stain EM analysis of purified VCP-AT3 particles indicate an enrichment of VCP hexamers, which is in contrast with the loss of hexamers from co-IPs of native ASPL (Figure 1). In order to investigate whether AT3 is sufficient to interact with VCP, we generated an in vitro reconstitution system using recombinantly purified His-tagged VCP, AT3 and ASPL. The components were combined and analyzed by size exclusion chromatography, which demonstrated that VCP forms a large complex in the presence of AT3 but not ASPL. Purified complexes were imaged by cryo-EM and subsequent reconstructions reveal novel densities that are putatively ascribed to AT3 (Figure 2). Ongoing efforts are underway to determine higher-resolution structures, which will be critical to resolve how AT3 drives transcription mis-regulation and provide a structural framework for ASPS treatment. 


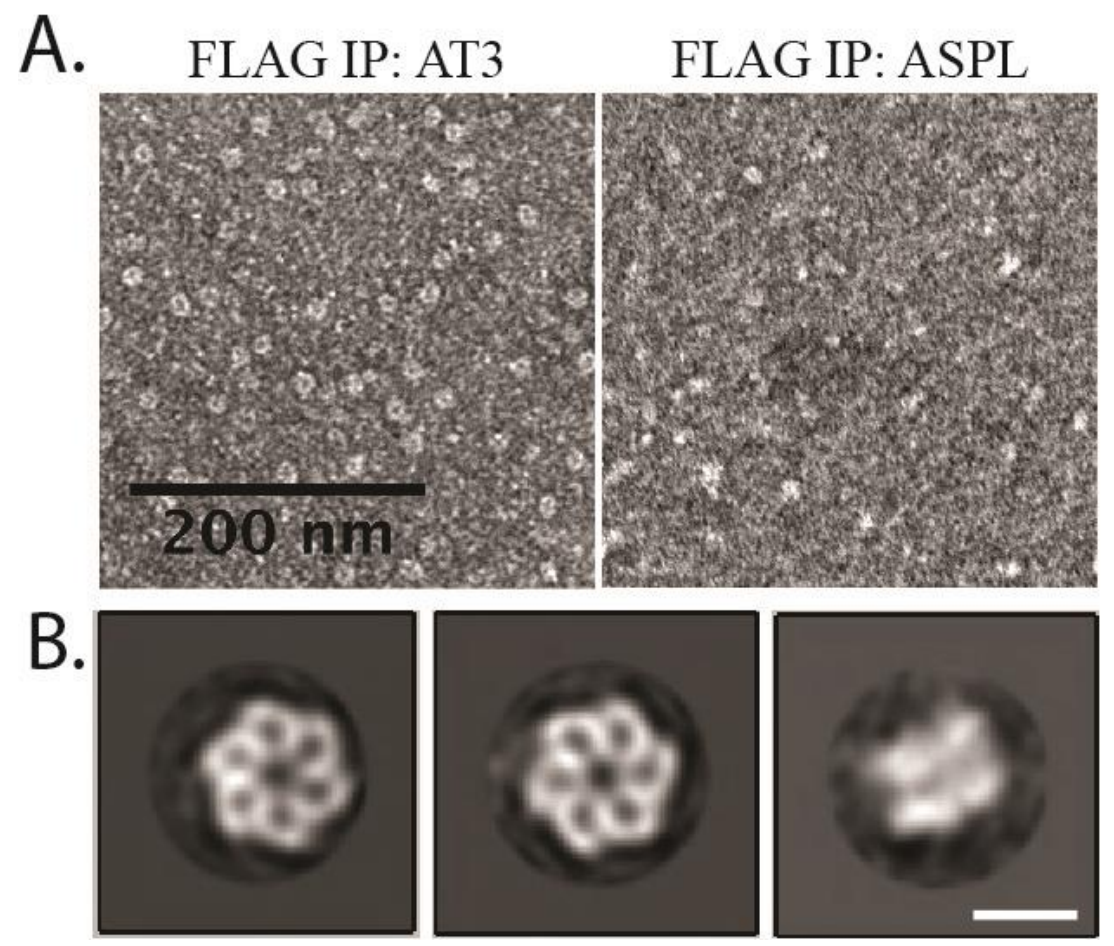

Figure 1. (A): Negative stain TEM micrographs of FLAG-IP eluates after AT3 or ASPSCR1 overexpression in HEK293T cells (scale bars $=200 \mathrm{~nm}$ ). $(\mathrm{B})$ : Reference-free 2D class averages of negatively stained particles recovered from FLAG-AT3 co-IP reveal intact VCP hexameric assemblies. Left and middle, VCP top views (3620 and 2774 particles in each class); Right, VCP side view (182 particles). Scale bar $=20 \mathrm{~nm}$.

A.

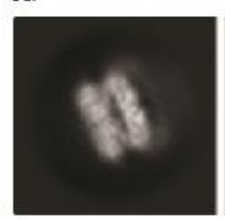

C.
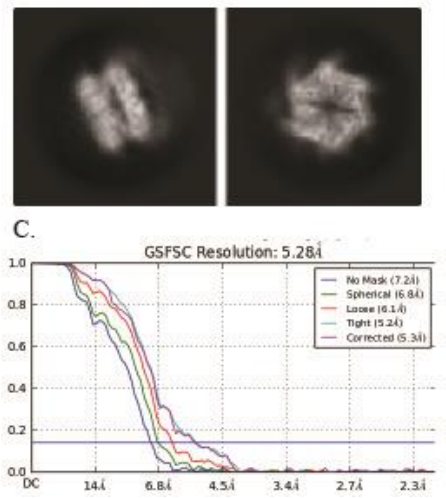

B.

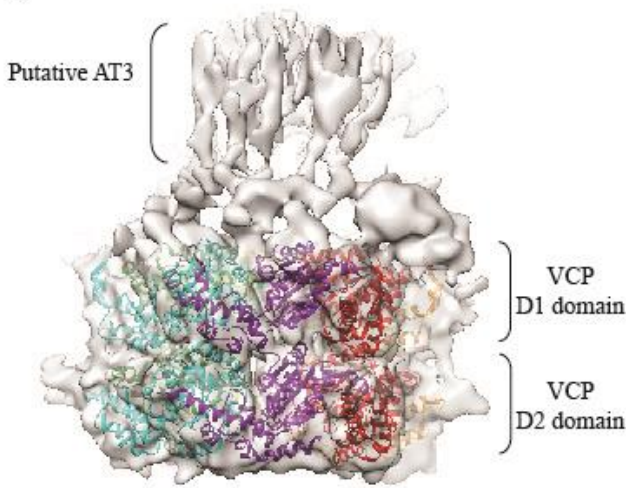

Figure 2. (A) Subset of representative, reference-free 2D class averages. (B) Reconstruction of recombinant AT3/VCP complex. (C) Gold-standard FSC plots.

\section{References}

[1] Cooney, I., etc. (2019) Structure of the Cdc48 segregase in the act of unfolding an authentic substrate, Science 365, 502-505.

[2] Xia, D., etc. (2016) Structure and function of the AAA+ ATPase p97/Cdc48p, Gene 583, 64-77.

[3] Meyer, H., etc. (2014) The VCP/p97 system at a glance: connecting cellular function to disease pathogenesis, Journal of cell science 127, 3877-3883. 
[4] Haines, D. S. (2010) p97-containing complexes in proliferation control and cancer: emerging culprits or guilt by association?, Genes \& cancer 1, 753-763.

[5] Orme, C. M., etc. (2012) The ubiquitin regulatory X (UBX) domain-containing protein TUG regulates the p97 ATPase and resides at the endoplasmic reticulum-golgi intermediate compartment, The Journal of biological chemistry 287, 6679-6692.

[6] Arumughan, A., etc. (2016) Quantitative interaction mapping reveals an extended UBX domain in ASPL that disrupts functional p97 hexamers, Nature communications 7, 13047.

[7] Ladanyi, M., etc. (2001) The der(17)t $(\mathrm{X} ; 17)(\mathrm{p} 11 ; \mathrm{q} 25)$ of human alveolar soft part sarcoma fuses the TFE3 transcription factor gene to ASPL, a novel gene at 17q25, Oncogene 20, 48-57.

[8] Joyama, S., etc. (1999) Chromosome rearrangement at 17q25 and xp11.2 in alveolar soft-part sarcoma: A case report and review of the literature, Cancer 86, 1246-1250.

[9] Vistica, D. T., etc. (2008) Immunohistochemical discrimination between the ASPL-TFE3 fusion proteins of alveolar soft part sarcoma, Journal of pediatric hematology/oncology 30, 46-52.

[10] Brady, O. A., etc. (2018) The transcription factors TFE3 and TFEB amplify p53 dependent transcriptional programs in response to DNA damage, eLife 7. [11] Tsuda, M., etc. (2007) TFE3 fusions activate MET signaling by transcriptional up-regulation, defining another class of tumors as candidates for therapeutic MET inhibition, Cancer research 67, 919-929.

[11] Tsuda, M., etc. (2007) TFE3 fusions activate MET signaling by transcriptional up- regulation, defining another class of tumors as candidates for therapeutic MET inhibition, Cancer research 67, 919929. 